\title{
Hormetic Effects of Flusilazole Preconditioning on Mycelial Growth and Virulence of Sclerotinia sclerotiorum
}

\author{
Xiaoming Lu, Shun He, Hongju Ma, Jianhong Li, and Fuxing Zhu, ${ }^{\dagger}$ College of Plant Science and Technology, Huazhong Agricultural \\ University, Wuhan, 430070, China
}

\begin{abstract}
Hormetic effects of fungicides are highly relevant to fungicide applications and management of plant-pathogenic fungi. Preconditioning (i.e., early exposure to relatively low doses of a toxicant) is a special form of hormesis, and fungicide preconditioning of phytopathogenic fungi is inevitable in the field. The present study showed that spraying the demethylation inhibitor (DMI) fungicide flusilazole at $0.1 \mu \mathrm{g} / \mathrm{ml}$ had stimulatory effects on the virulence of Sclerotinia sclerotiorum inoculated at 1 and $24 \mathrm{~h}$ after spraying. Flusilazole sprayed at $10 \mu \mathrm{g} / \mathrm{ml}$ showed inhibitory effects on the virulence of S. sclerotiorum inoculated during the first 3 days after spraying. Inoculations on the 5th, 7th, and 10th day after spraying did not show any significant inhibitory or stimulatory effects on the virulence. After growing for 2 days on potato dextrose agar (PDA) amended with flusilazole at a dose range from 0.0005 to $0.25 \mu \mathrm{g} / \mathrm{ml}$ as preconditioning treatments, mycelia were transferred onto PDA without fungicide and subsequent mycelial growth was slower than the nonpreconditioned control. However, after the preconditioned colonies were transferred onto PDA supplemented with flusilazole

at $0.2 \mu \mathrm{g} / \mathrm{ml}$, percent stimulations of mycelia growth compared with the control had a parabolic shape across the preconditioning flusilazole concentration range. Similarly, the mycelial growth of the preconditioned mycelial plugs on PDA amended with other DMI fungicides (prochloraz or tebuconazole) also showed a typical hormetic response, whereas mycelial growth on PDA amended with carbendazim or dimethachlone was inhibited in a dosedependent manner. Preconditioning S. sclerotiorum with flusilazole on rapeseed plants elicited virulence stimulations in a dose-dependent manner similar to those on mycelial growth on PDA. After disease lesions developed on rapeseed leaves sprayed with flusilazole as the preconditioning treatment were inoculated onto rapeseed plants, virulence was inhibited on leaves without fungicide or sprayed with carbendazim or dimethachlone compared with the nonpreconditioned control, whereas virulence was stimulated on leaves sprayed with flusilazole, prochloraz, or tebuconazole, and the maximum percent stimulation was $10.2 \%$. These results will advance our understanding of hormetic effects of fungicides and of preconditioning hormesis in particular.
\end{abstract}

Due to different distributions and penetrations through crop canopies, spraying a fungicide in the field will inevitably lead to exposure of plant pathogens to sublethal doses of the applied fungicide (Flores and Garzón 2013). Exposure of pathogens to sublethal doses of a fungicide may result in increased virulence to crops, and this phenomenon belongs to a common biological concept called hormesis (Di et al. 2015). Hormesis is a kind of dose-response relationship characterized by low-dose stimulation and high-dose inhibition (Calabrese 2015a). Hormesis is a fundamental and general biology concept, and hormetic responses have been reported in a wide range of biological models and across a large variety of chemical classes (Calabrese 2015a). Up to 2011 , approximately 9,000 cases of hormesis had been included in a relational retrieval hormesis database (Calabrese and Blain 2011). Qualitative and quantitative features of hormesis such as frequency, stimulation amplitude, and dose range of chemicals have been thoroughly reviewed, mainly by Calabrese (2013, 2015a,b, 2016a,b) and Calabrese and Mattson (2017). Stimulatory effects of sublethal doses of fungicides on mycelial growth and virulence of bacteria, oomycetes (Flores and Garzón 2013; Garzón et al. 2011), and fungi (Baraldi et al. 2003; Di et al. 2015, 2016a,b) have been reported. These studies primarily focused on the stimulatory effects of low doses of fungicides on plant pathogens, in vitro or in planta. However, in the field, fungicides are applied at relatively high doses, and the pathogen may arrive at the surface of crops after a period of time. The time profile of the effects of fungicides on the virulence of plant pathogens is not yet clear and, thus, merits studies.

${ }^{\dagger}$ Corresponding author: F. Zhu, E-mail: zhufuxing@mail.hzau.edu.cn

$\mathrm{X}$. Lu and $\mathrm{S}$. He contributed equally to this work.

Funding: This study was supported by the National Natural Science Foundation of China (31371964)

Accepted for publication 21 January 2018.

(c) 2018 The American Phytopathological Society
Preconditioning is a specific type of hormesis (Calabrese 2016a,b). Preconditioning hormesis refers to the phenomenon where early exposure to low to moderate doses of a toxicant or stress will lower the damage caused by subsequent relatively high doses of related or unrelated toxicants or stresses. Fungicide preconditioning has significant implications for plant pathogen management strategies. In the field, plant pathogens exposed to moderate doses of a fungicide may be partially inhibited but not completely eradicated. The inhibited (i.e., preconditioned) spores and mycelia will infect crop plants some time later, and the effects of earlier fungicide exposure on later virulence are not clear. In addition, a fungicide is usually sprayed twice with a time interval of about 2 weeks in a growing season. It is highly possible that a pathogen may have been preconditioned by the first application when exposed to the second spraying. Although preconditioning hormesis has been extensively studied and reviewed recently (Calabrese 2016a,b; Calabrese et al. 2007), to the best of our knowledge, no systematic studies have been reported thus far on the quantitative features of fungicide preconditioning. Sclerotinia sclerotiorum is a devastating necrotrophic plantpathogenic fungus with a wide host range and it often causes heavy yield losses to many economically important crops (Boland and Hall 1994; Bolton et al. 2006; USDA 2016; Wang et al. 2014). The sterol demethylation inhibitor (DMI) fungicide flusilazole is a broad-spectrum fungicide with high preventative and curative efficacies against $S$. sclerotiorum (Lu et al. 2015), and stimulatory effects of low doses of flusilazole on the virulence of $S$. sclerotiorum have been reported (Lu et al. 2018). The objectives of the present study were to (i) assess the effects of different concentrations of flusilazole on the virulence of $S$. sclerotiorum mycelial plugs inoculated at different time points after spraying the fungicide, (ii) evaluate preconditioning hormetic effects of flusilazole on mycelial growth of $S$. sclerotiorum on potato dextrose agar (PDA), and (iii) determine preconditioning hormetic effects of flusilazole on the virulence of $S$. sclerotiorum on rapeseed plants.

\section{Materials and Methods}

Collection and preservation of $\boldsymbol{S}$. sclerotiorum isolates. Two S. sclerotiorum isolates, HN-24 and GS-7, which had been used in previous studies on toxic and hormetic effects of flusilazole, were collected in 2012 from rapeseed fields of Hunan and Gansu provinces, respectively 
(Lu et al. 2015, 2018). Mature sclerotia were collected from diseased rapeseed plants and preserved at $4{ }^{\circ} \mathrm{C}$ for long term storage. Both isolates were sensitive to flusilazole, and the effective concentrations at which mycelial growth was inhibited by $50 \%$ were 0.085 and $0.104 \mu \mathrm{g} / \mathrm{ml}$ for isolates HN-24 and GS-7, respectively (Lu et al. 2015).

Fungicide. Technical grade flusilazole (96.4\% active ingredient [a.i.], Hubei Kang Bao Tai Fine-Chemical Co. Ltd.), prochloraz $(97.0 \%$ a.i., Hubei Kang Bao Tai Fine-Chemical Co. Ltd.), tebuconazole (96.0\% a.i., Jiangsu Huanghai Agro-Chemical Co. Ltd.), dimethachlone (96.0\% a.i., Zhejiang Weizhou Pesticide Co. Ltd.), and carbendazim (98.1\% a.i., Tian Jin Jin Bei Fine-Chemical Co. Ltd.) were stored at $4^{\circ} \mathrm{C}$. Stock solutions of flusilazole, prochloraz, and tebuconazole at $1,000 \mu \mathrm{g} / \mathrm{ml}$ were prepared by dissolving each of these fungicides in pure acetone. Dimethachlone was dissolved in methanol, and carbendazim was dissolved in hydrochloric acid $(\mathrm{HCl})$ at $0.1 \mathrm{~mol} /$ liter to prepare $1,000 \mu \mathrm{g} / \mathrm{ml}$ stock solutions. Stock solutions were kept at $4^{\circ} \mathrm{C}$ for no longer than 2 weeks before being diluted for subsequent experiments.

Effects of flusilazole on the virulence of $S$. sclerotiorum inoculated at different time points after spraying on potted rapeseed plants. The procedure for assessing the stimulatory effects of sprayed flusilazole on virulence was according to Di et al. (2016a) and $\mathrm{Lu}$ et al. (2018). The flusilazole stock solution was serially diluted with $0.1 \%$ Triton $\mathrm{X}-100$ in water to final concentrations of $100,10,1$, and $0.1 \mu \mathrm{g} / \mathrm{ml}$. Flusilazole dilutions were sprayed with a handheld sprayer $(800 \mathrm{ml}$ in volume) until runoff on potted 4week-old rapeseed plants growing in the greenhouse. Rapeseed plants sprayed with $0.1 \%$ Triton X-100 in water were used as the nontreated control. After being air dried for $1 \mathrm{~h}$, three rapeseed plants (two leaves per plant) were inoculated on the adaxial leaf surface with inverted 2-day-old mycelial plugs ( $5 \mathrm{~mm}$ in diameter). In the same way, rapeseed plants were inoculated with mycelial plugs on the 1st, 3rd, 5th, 7 th, and 10th day after being sprayed with flusilazole. The inoculated plants were incubated at $23^{\circ} \mathrm{C}$ with a photoperiod of $16 \mathrm{~h}$ of light and $8 \mathrm{~h}$ of darkness and with relative humidity maintained at about $85 \%$. After $48 \mathrm{~h}$ of incubation, lesion diameters were measured twice at right angles. All experiments in the study were conducted in triplicate and repeated independently at least twice.

Effects of flusilazole preconditioning on mycelial growth of S. sclerotiorum on PDA. The procedure was according to Zhou et al. (2014), with minor modifications. Mycelial plugs were inoculated on PDA amended with flusilazole at final concentrations of 0.0005 , $0.001,0.0025,0.005,0.01,0.02,0.04,0.08,0.16,0.25$, and $0.32 \mu \mathrm{g} / \mathrm{ml}$. The PDA plates were incubated at $23^{\circ} \mathrm{C}$ in the dark for $48 \mathrm{~h}$ as the preconditioning treatment. Mycelial plugs cut from the fresh margins of the preconditioned colonies were transferred onto PDA without fungicide and onto PDA supplemented with flusilazole at a final concentration of $0.2 \mu \mathrm{g} / \mathrm{ml}$. Mycelial plugs without flusilazole preconditioning treatment were inoculated on PDA without flusilazole and on PDA amended with flusilazole at $0.2 \mu \mathrm{g} / \mathrm{ml}$ as the nonpreconditioned controls. The diameter of each colony was measured twice in two perpendicular directions after $48 \mathrm{~h}$ of incubation.

Effects of flusilazole preconditioning on mycelial growth on PDA amended with other fungicides. The preconditioning treatment was similar to that described above in the last section. Preliminary experiments with a wider flusilazole dosage range indicated that preconditioning with flusilazole at a concentration range of 0.005 to $0.16 \mu \mathrm{g} / \mathrm{ml}$ had detectable stimulatory effects on mycelial growth on PDA amended with fungicides compared with the nonpreconditioned control. Therefore, mycelia were grown for $48 \mathrm{~h}$ on PDA supplemented with flusilazole at 0.005 to $0.32 \mu \mathrm{g} / \mathrm{ml}$ as the preconditioning treatments. Mycelial plugs cut from fresh margins of the preconditioned colonies were transferred onto PDA amended with either prochloraz at $0.1 \mu \mathrm{g} / \mathrm{ml}$, tebuconazole at $0.1 \mu \mathrm{g} / \mathrm{ml}$, carbendazim at $0.1 \mu \mathrm{g} / \mathrm{ml}$, or dimethachlone at $0.2 \mu \mathrm{g} / \mathrm{ml}$. The concentrations of these fungicides were employed based on preliminary experiments showing that these concentrations caused similar inhibitions on mycelial growth for those mycelial plugs without being preconditioned. The diameter of each colony was measured twice at right angles after $48 \mathrm{~h}$ of incubation. Mycelial plugs without being preconditioned were used as the nonpreconditioned control.
Effects of flusilazole preconditioning on the virulence of S. sclerotiorum on rapeseed plants. A stock solution of flusilazole was serially diluted with $0.1 \%$ Triton X-100 in water to final concentrations of 120, 90, 60, 40, 20, 10, and $5 \mu \mathrm{g} / \mathrm{ml}$. Potted 4-week-old rapeseed plants were sprayed with the flusilazole dilutions as the preconditioning treatment with a handheld sprayer, as described above. After being air dried for about $1 \mathrm{~h}$, the rapeseed plants were inoculated with inverted fresh mycelial plugs on the adaxial leaf surface and incubated at $23^{\circ} \mathrm{C}$ for $48 \mathrm{~h}$ with relative humidity maintained at about $85 \%$. Then, freshly developed disease lesions were detached from leaves with a pair of forceps and inoculated onto the adaxial leaf surface of 4 -week-old rapeseed plants sprayed with $0.1 \%$ Triton $\mathrm{X}-100$ in water or with flusilazole at $15 \mu \mathrm{g} / \mathrm{ml}$. After being incubated at $23^{\circ} \mathrm{C}$ for $48 \mathrm{~h}$, lesion diameters were measured twice at right angles. Lesion inocula without being preconditioned with flusilazole were used as the nonpreconditioned control.

Effects of flusilazole preconditioning on the virulence of S. sclerotiorum on rapeseed plants treated with other fungicides. The preconditioning treatment procedure was the same as described above. The freshly developed disease lesions preconditioned with flusilazole were detached from leaves and inoculated on the adaxial leaf surface of 4-week-old rapeseed plants sprayed with either prochloraz at $15 \mu \mathrm{g} / \mathrm{ml}$, tebuconazole at $25 \mu \mathrm{g} / \mathrm{ml}$, dimethachlone at $10 \mu \mathrm{g} / \mathrm{ml}$, or carbendazim at $30 \mu \mathrm{g} / \mathrm{ml}$. The concentrations of these fungicides were employed based on preliminary experiments showing that these concentrations caused similar inhibitions on the virulence of $S$. sclerotiorum without being preconditioned. After being incubated at $23^{\circ} \mathrm{C}$ for $48 \mathrm{~h}$, lesion diameters were measured twice in perpendicular directions. Lesion inocula without being preconditioned with flusilazole were used as the nonpreconditioned control.

Data processing and analysis. Percent stimulations of virulence were calculated by the following formula: Percent stimulation $(\%)=$ [(lesion diameter of the treated - lesion diameter of the control)/ lesion diameter of the control] $\times 100 \%$. Percent stimulations of mycelial growth were calculated with a similar formula, only with mycelial colony diameter being used to substitute for lesion diameter. Arcsine square-root transformation was performed on the percent stimulation data before further statistical analysis. Data from different repetitions of the same experiment were pooled together because differences among repetitions were not significant. The multiple comparison procedure Dunnett' test $(\alpha=0.05)$ in the statistical software SPSS (Statistical Product and Service Solutions, ver. 21.0; SPSS Inc.) was employed to assess the statistical significance of percent stimulations of each treatment compared with the nontreated control. Curve modeling was performed according to Deng et al. (2001) with the second-order polynomial (quadratic) procedure in the software Microsoft Excel (ver. 2010; Microsoft Corporation).

\section{Results}

Stimulatory effects of flusilazole on the virulence of $S$. sclerotiorum inoculated at different time points after spraying on potted rapeseed plants. Compared with the nontreated control, flusilazole at low doses of 0.1 and $1 \mu \mathrm{g} / \mathrm{ml}$ exhibited stimulatory effects on the virulence of $S$. sclerotiorum inoculated from $1 \mathrm{~h}$ to the fifth day after spraying the fungicide (Fig. 1). For both isolates tested, flusilazole at $0.1 \mu \mathrm{g} / \mathrm{ml}$ had significant $(P<0.05)$ stimulatory effects on the virulence of mycelial plugs inoculated at 1 and $24 \mathrm{~h}$ after spraying. Flusilazole at $1 \mu \mathrm{g} / \mathrm{ml}$ had significant stimulatory effects on the virulence of isolate $\mathrm{HN}-24$ inoculated at $1 \mathrm{~h}$ after spraying. For isolate GS-7, flusilazole at $1 \mu \mathrm{g} / \mathrm{ml}$ showed a maximum stimulatory effect for the inoculations on the 3rd day after spraying, whereas inoculations at $1 \mathrm{~h}$ and on the $1 \mathrm{st}, 7 \mathrm{th}$, and 10th day after spraying did not show significant virulence stimulations. Flusilazole sprayed at $10 \mu \mathrm{g} / \mathrm{ml}$ showed significant $(P<0.05)$ inhibitory effects on the virulence of $S$. sclerotiorum inoculated during the first 3 days after spraying. Flusilazole at $10 \mu \mathrm{g} / \mathrm{ml}$ did not have significant inhibitory or stimulatory effects on the virulence of either of the two isolates inoculated on the 5 th, 7 th, and 10th day after spraying. Flusilazole at $100 \mu \mathrm{g} / \mathrm{ml}$ showed 82.6 and $85.1 \%$ of inhibition of virulence for the two isolates HN-24 and GS-7, respectively, inoculated at $1 \mathrm{~h}$ after 
the spraying of the fungicide and 22.6 and $42.6 \%$ of inhibition for the two isolates, respectively, inoculated on the 10th day after the spraying (data not shown).

Effects of flusilazole preconditioning on mycelial growth of S. sclerotiorum on PDA. After growing for $48 \mathrm{~h}$ on PDA amended with different concentrations of flusilazole as preconditioning treatment, the mycelia were transferred onto PDA without fungicide, and the mycelial growth decreased in a dose-dependent manner compared with the nonpreconditioned control (Fig. 2). Preconditioning flusilazole at a concentration range of 0.0005 to $0.32 \mu \mathrm{g} / \mathrm{ml}$ in PDA caused mycelial growth inhibited by 2.2 to $40.9 \%$ for the first generation of isolate $\mathrm{HN}-24$. After the preconditioned colonies were transferred onto PDA without fungicide, mycelial growths were still inhibited by 0.8 to $18.0 \%$ for the second generation compared with the nonpreconditioned control. However, after the preconditioned mycelial colonies were transferred onto PDA amended with flusilazole at $0.2 \mu \mathrm{g} / \mathrm{ml}$, mycelial growths were inhibited compared with that on PDA without flusilazole. However, compared with the nonpreconditioned control, the mycelial growths on PDA amended with flusilazole at $0.2 \mu \mathrm{g} / \mathrm{ml}$ showed a typical hormetic dose-response pattern. The preconditioning flusilazole concentration range with significant stimulatory effects was
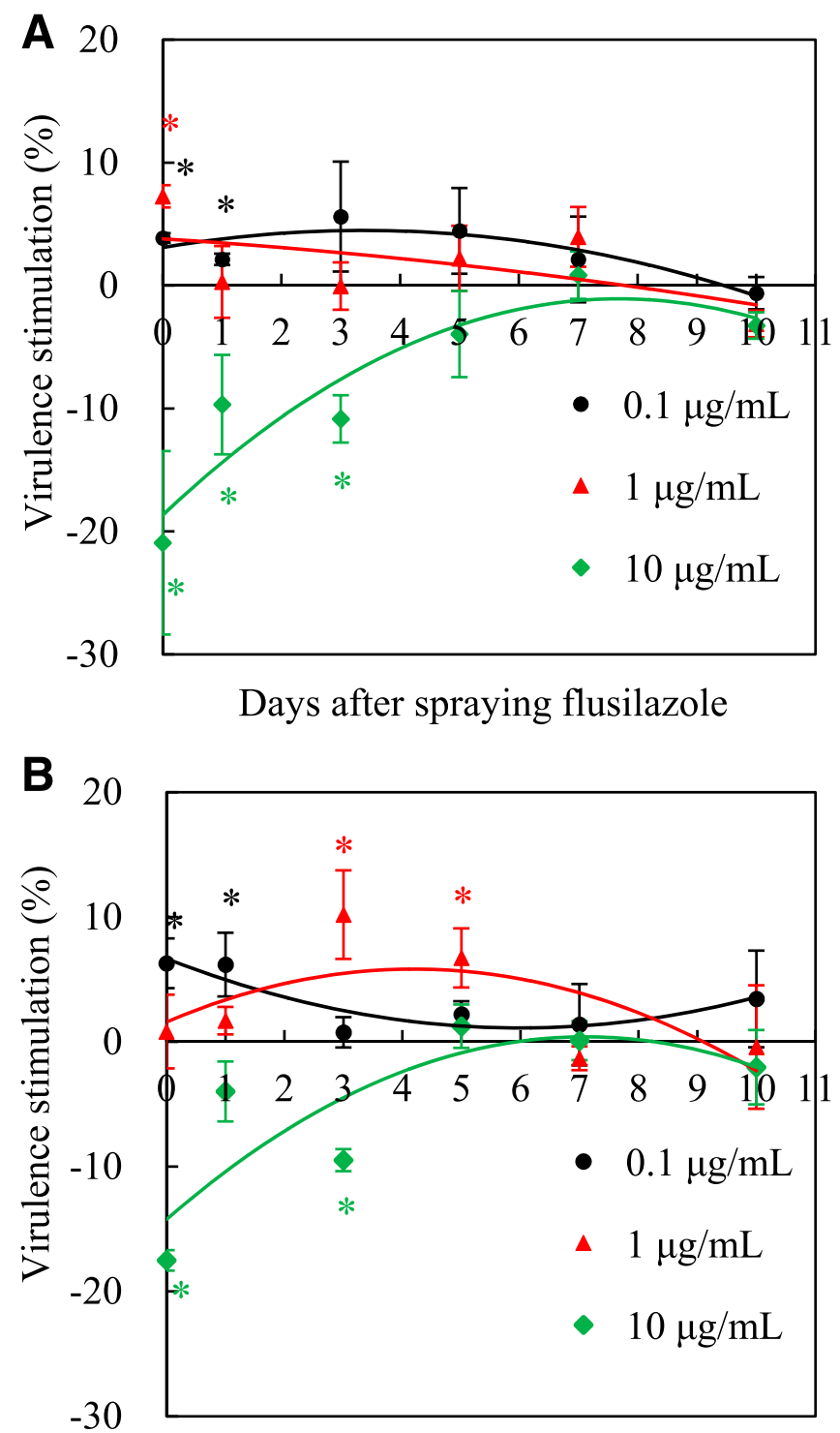

Days after spraying flusilazole

Fig. 1. Virulence stimulations for Sclerotinia sclerotiorum isolates $\mathbf{A}, \mathrm{HN}-24$ and $\mathbf{B}$, GS-7 inoculated at different days after spraying flusilazole on rapeseed leaves. Error bar denotes the standard error of the mean of three repetitions. Asterisk denotes significant difference from the control at $\alpha=0.05$. from 0.005 to $0.08 \mu \mathrm{g} / \mathrm{ml}$, and the maximum stimulation magnitude was $8.7 \%$ for isolate $\mathrm{HN}-24$. The preconditioning flusilazole concentration range with significant stimulatory effects was from 0.0025 to $0.04 \mu \mathrm{g} / \mathrm{ml}$ for isolate GS-7, and the maximum stimulation magnitude was $9.9 \%$.

Effects of flusilazole preconditioning on mycelial growth on PDA amended with other fungicides. After the preconditioned mycelial colonies were transferred onto PDA amended with either prochloraz, tebuconazole, carbendazim, or dimethachlone, mycelial growth exhibited two distinct patterns of responses (Fig. 3). On PDA amended with prochloraz or tebuconazole, which has the same mode of action as flusilazole, mycelial growth displayed a similar response pattern similar to that on PDA supplemented with flusilazole, only with higher preconditioning flusilazole concentrations for the stimulatory effects. The maximum stimulation magnitude was a little lower than that on PDA amended with flusilazole. However, mycelial growths of the preconditioned mycelial plugs on PDA amended with carbendazim or dimethachlone were inhibited compared with the nonpreconditioned control.

Effects of flusilazole preconditioning on the virulence of S. sclerotiorum on rapeseed plants. Mycelial plugs were inoculated on leaves of rapeseed plants sprayed with different concentrations of flusilazole as the preconditioning treatments, and lesion developments were inhibited by 2.6 to $87.1 \%$ for isolates $\mathrm{HN}-24$ when the
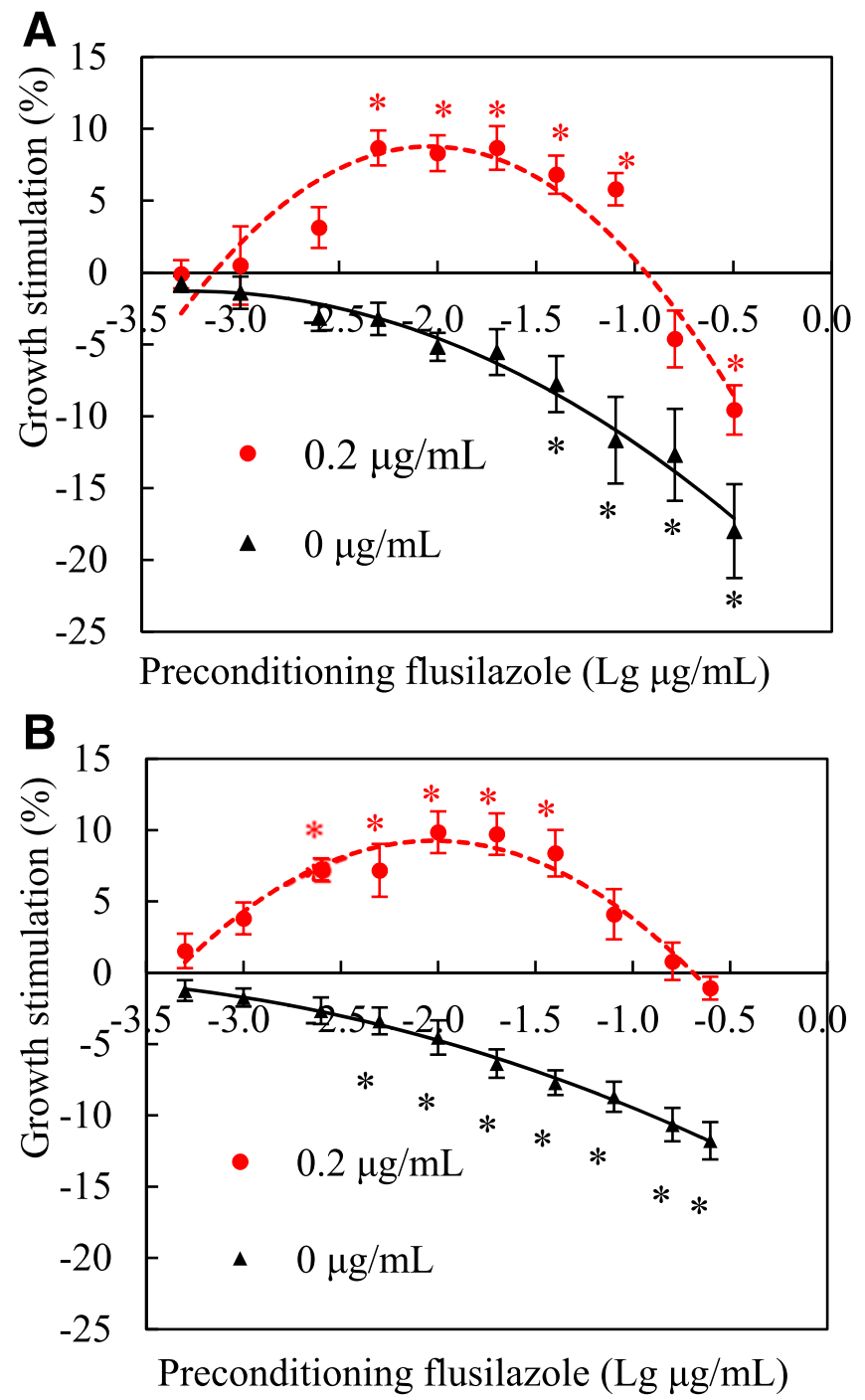

Fig. 2. Effects of preconditioning with flusilazole on mycelial growth of Sclerotinia sclerotiorum isolates A, HN-24 and B, GS-7 on potato dextrose agar with or without flusilazole. The diameter of each colony was measured after growing for $48 \mathrm{~h}$. Error bar denotes the standard error of the mean of four repetitions. Asterisk denotes significant difference from the control at $\alpha=0.05$. 
preconditioning flusilazole concentrations were from 5 to $120 \mu \mathrm{g} / \mathrm{ml}$. For isolate GS-7, flusilazole at 5 to $80 \mu \mathrm{g} / \mathrm{ml}$ caused lesion developments to be inhibited by 4.4 to $70.3 \%$ (data not shown). After freshly developed lesions were detached and inoculated on healthy leaves without fungicide, lesion developments were inhibited for the preconditioned inocula compared with nonpreconditioned control. The highest percent inhibitions were 13.9 and $14.2 \%$ for isolates $\mathrm{HN}$ 24 and GS-7, respectively, and the corresponding preconditioning concentrations were 120 and $60 \mu \mathrm{g} / \mathrm{ml}$, respectively (Fig. 4). However, when the preconditioned lesions were inoculated on leaves sprayed with flusilazole at $15 \mu \mathrm{g} / \mathrm{ml}$, lesion developments exhibited a typical, biphasic hormetic dose-response pattern. Compared with the nonpreconditioned control, preconditioning treatments with flusilazole at 20,40 , and $60 \mu \mathrm{g} / \mathrm{ml}$ showed significant $(P<0.05)$ stimulatory effects on lesion development for isolate $\mathrm{HN}-24$. The maximum percent stimulations for lesion development on flusilazole treated rapeseed leaves were 17.8 and $10.4 \%$ for isolates $\mathrm{HN}-24$ and GS-7, respectively.

Effects of flusilazole preconditioning on the virulence of $S$. sclerotiorum on rapeseed plants treated with other fungicides.

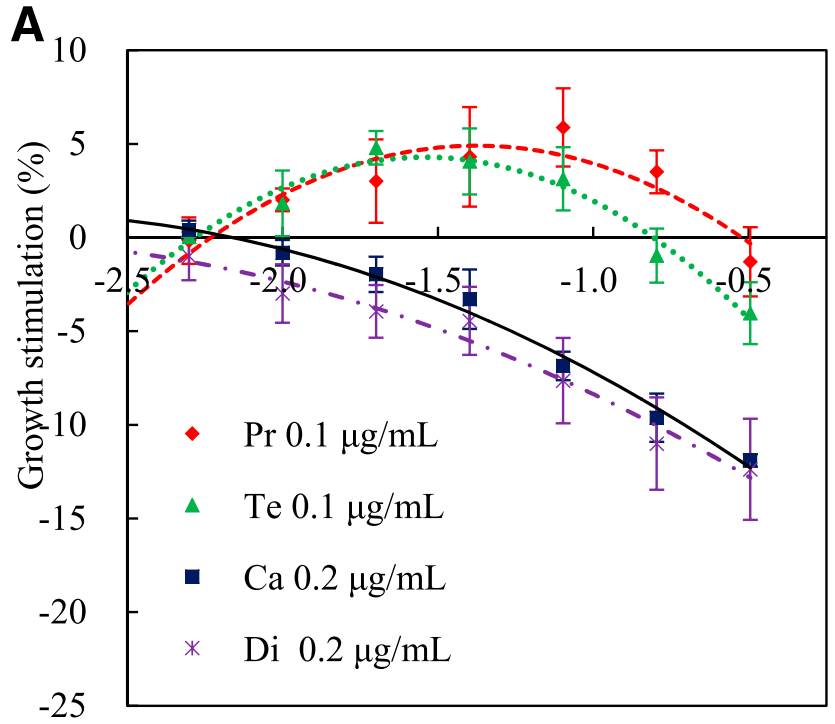

Preconditioning flusilazole $(\mathrm{Lg} \mu \mathrm{g} / \mathrm{mL})$

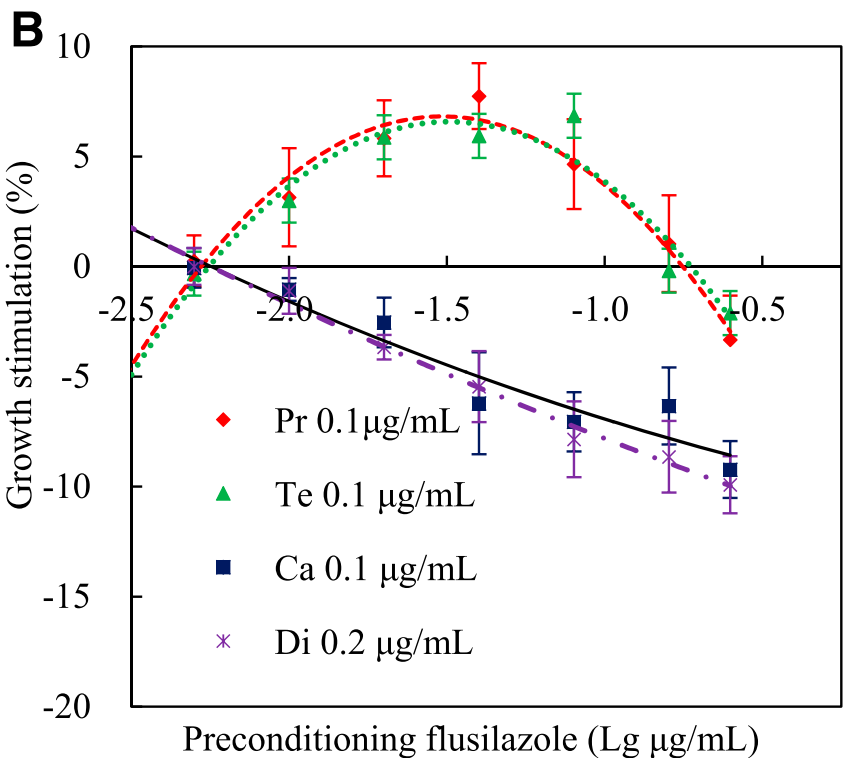

Fig. 3. Effects of preconditioning with flusilazole on mycelial growth of Sclerotinia sclerotiorum isolates A, HN-24 and B, GS-7 on potato dextrose agar amended with prochloraz $(\mathrm{Pr})$, tebuconazole $(\mathrm{Te})$, carbendazim $(\mathrm{Ca})$, or dimethachlone (Di). Error bar denotes the standard error of the mean. Data are mean values of four replicates.
After the preconditioned lesions were inoculated on rapeseed leaves sprayed with other fungicides, disease lesions developed in a dosedependent manner similar to that of the mycelial growth on PDA amended with fungicides (Fig. 5). After the preconditioned disease lesions were inoculated on rapeseed leaves sprayed with ether flusilazole, prochloraz, or tebuconazole, disease lesions developed in a similar, hormetic dose-response pattern (i.e., the preconditioned inocula exhibited stimulated disease developments compared with the nonpreconditioned control). The maximum percent stimulations for isolates HN-24 and GS-7 were 10.2 and 9.7\%, occurring on rapeseed leaves sprayed with prochloraz when the concentrations of preconditioning flusilazole were 40 and $20 \mu \mathrm{g} / \mathrm{ml}$, respectively. However, on rapeseed leaves sprayed with carbendazim or dimethachlone, lesion developments were progressively inhibited compared with the nonpreconditioned control.

\section{Discussion}

The present study demonstrated that flusilazole sprayed at low doses had significant stimulatory effects on the virulence of $S$. sclerotiorum inoculated at the first 3 days after spraying. This was not a surprise because the hormetic effects of flusilazole on the virulence of $S$. sclerotiorum have been reported in our previous studies ( $\mathrm{Lu}$
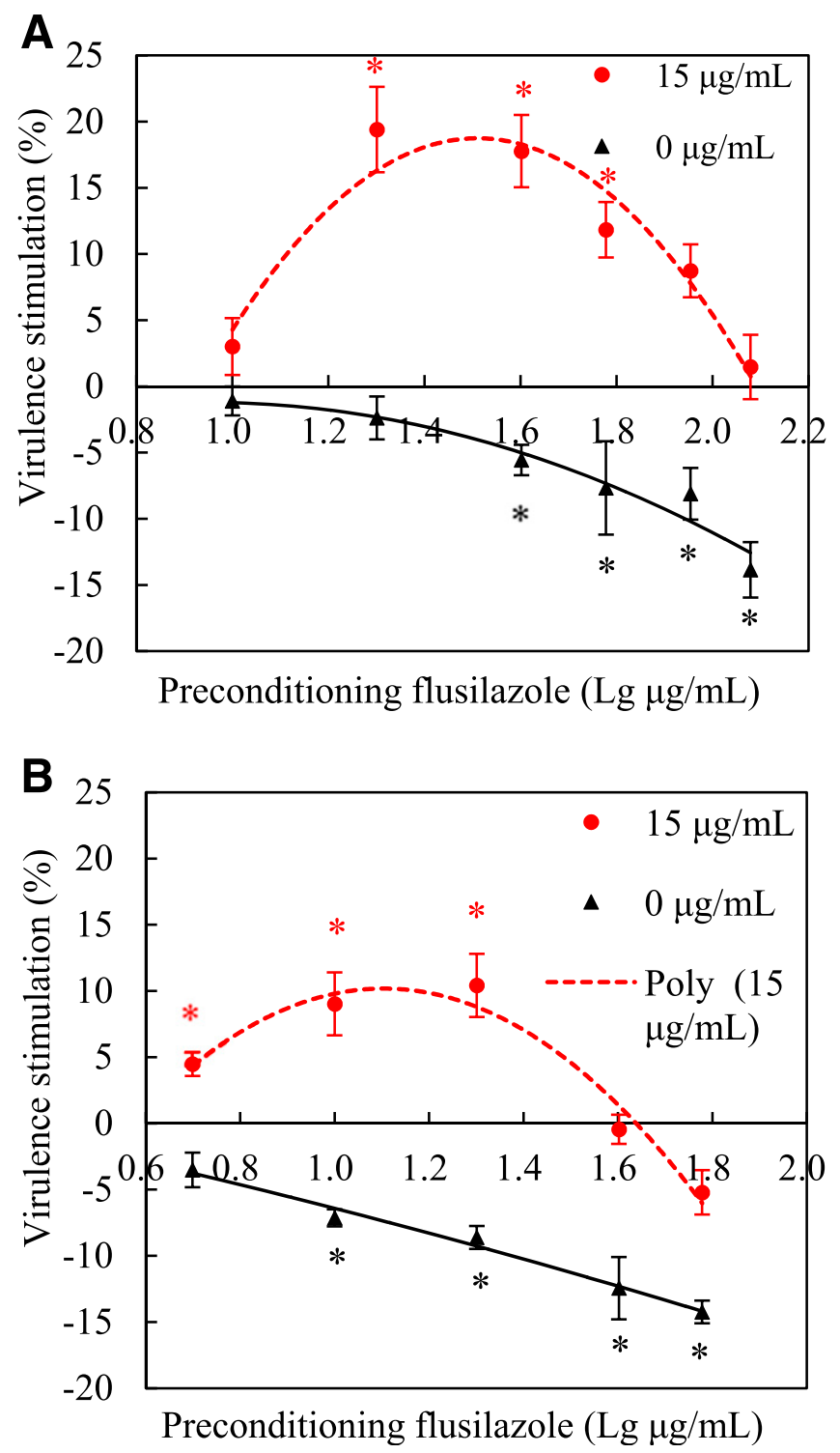

Fig. 4. Effects of preconditioning with flusilazole on virulence of Sclerotinia sclerotiorum isolates A, HN-24 and B, GS-7 on rapeseed leaves treated with or without flusilazole. Error bar denotes the standard error of the mean of four repetitions. Asterisk denotes significant difference from the control at $\alpha=0.05$. 
et al. 2018). Flusilazole is recommended to be sprayed at around 50 to $100 \mu \mathrm{g} / \mathrm{ml}$ to control a variety of fungal pathogens in the field. In the present study, flusilazole sprayed at $100 \mu \mathrm{g} / \mathrm{ml}$ did not show any signs of stimulatory effects on the virulence of $S$. sclerotiorum inoculated several days after spraying when the concentrations of flusilazole on the leaves were presumed to be low due to degradation. Flusilazole sprayed at $10 \mu \mathrm{g} / \mathrm{ml}$ exhibited significant inhibitory effects on the virulence of $S$. sclerotiorum inoculated at the first 3 days after spraying. From the 5th to the 10th day, no significant stimulatory or inhibitory effects could be observed. Flusilazole decomposes quickly in the field, and the half-life time of flusilazole is approximately 4.2 to 7.8 and 6.3 to 8.4 days on apple and mandarin, respectively (Wang et al. 2013; Yu et al. 2011). The major reason for the failure of detecting the stimulatory effects on the 10th day after spraying may be due to the relatively short period of experiment time. After a longer period of time, stimulatory effects may be detected. In theory, the concentration of a fungicide sprayed at relatively high doses will decline quickly on crops and, after an appropriate time period, the fungicide may exhibit stimulatory effects on the virulence of plant pathogens. The stimulatory effects of a fungicide at different time points after application need further investigations.

When the inhibited mycelia grown on flusilazole-amended PDA were inoculated on PDA without fungicide, mycelial growth was still inhibited compared with the nonpreconditioned control. This result indicates that the inhibitory effects on mycelial growth of the first generation could extend to the next generation in the absence of the fungicide. However, after the preconditioned colonies were transferred onto PDA amended with flusilazole, mycelial growth was stimulated compared with the nonpreconditioned control. For the two isolates assayed, the maximum stimulation magnitude of mycelial growth was no more than $10 \%$. The concentration range of preconditioning flusilazole with growth stimulations was from 0.001 to $0.08 \mu \mathrm{g} / \mathrm{ml}$. This stimulation profile conformed well to the established biological concept hormesis. Preconditioning is a special form of hormesis (Calabrese 2016a,b). The present study showed that, compared with the nonpreconditioned control, the preconditioned mycelial plugs had increased growth rates not only on PDA amended with flusilazole but also on PDA amended with prochloraz or tebuconazole, indicating that preconditioning with flusilazole had hormetic effects for later exposure to flusilazole, prochloraz, or tebuconazole, which all belong to the same class of fungicides in terms of mode of action (MoA). On PDA amended with carbendazim or dimethachlone, the mycelial growth of the preconditioned plugs was significantly inhibited compared with the nonpreconditioned control. This phenomenon indicates that fungicide preconditioning effects seem to be MoA specific, and this has significant implications for understanding the mechanism of preconditioning hormesis.

The effects of preconditioning with flusilazole on disease lesion developments on rapeseed leaves were similar to the preconditioning effects on mycelial growth on PDA. The inhibitory effects of preconditioning with flusilazole on disease lesion developments persisted to the next generation disease developments on leaves without a fungicide. However, when the preconditioned disease lesions were inoculated on rapeseed leaves sprayed with flusilazole, disease lesions developed faster than the nonpreconditioned control. The stimulatory effects of preconditioning with flusilazole manifested themselves not only for subsequent exposure to flusilazole itself but also for exposure to prochloraz or tebuconazole. However, for subsequent exposure to dimethachlone or carbendazim, no stimulatory effects could be detected. These results have profound implications for fungicide applications in the field. Fungicide preconditioning may exert stimulatory effects on the virulence of pathogenic fungi, implying that spraying a fungicide at relatively high doses may be better than the splitting dosage statics. Preconditioning pathogenic fungi with a fungicide may increase the virulence of the fungi in the presence of the same fungicide or other fungicides with the same MoA as that of the preconditioning fungicide. These results imply that fungicides with the same MoA should not be used in succession lest preconditioning hormesis should occur. Considering the importance of preconditioning hormesis to fungicide application tactics, the temporal features of preconditioning effects merit further studies in the future.

It is interesting to note that there were considerable differences in stimulation amplitude for later exposure to different fungicides. For mycelia of the two isolates preconditioned with flusilazole, subsequent exposure to flusilazole exhibited the greatest growth stimulation compared with the nonpreconditioned control. Mycelial growth stimulations for subsequent exposure to either prochloraz or tebuconazole were slightly lower than that for exposure to flusilazole whereas, for exposure to dimethachlone and carbendazim, mycelial growth was inhibited rather than stimulated. Similarly, for the virulence of the two isolates preconditioned with flusilazole, subsequent exposure to flusilazole exhibited the highest stimulation, and exposure to either prochloraz or tebuconazole displayed slightly lower stimulations, whereas the virulence was inhibited for subsequent exposure to either carbendazim or dimethachlone. Although the concentrations of subsequent treatment fungicides were different, with flusilazole, carbendazim, and dimethachlone all at $0.2 \mu \mathrm{g} / \mathrm{ml}$ while prochloraz and tebuconazole both at $0.1 \mu \mathrm{g} / \mathrm{ml}$ for the mycelial growth assays, the inhibitory effects of these fungicides at the concentrations employed were similar in amplitude. These results indicate that the fungicide preconditioning effects were mainly related to the MoA of the fungicide used.

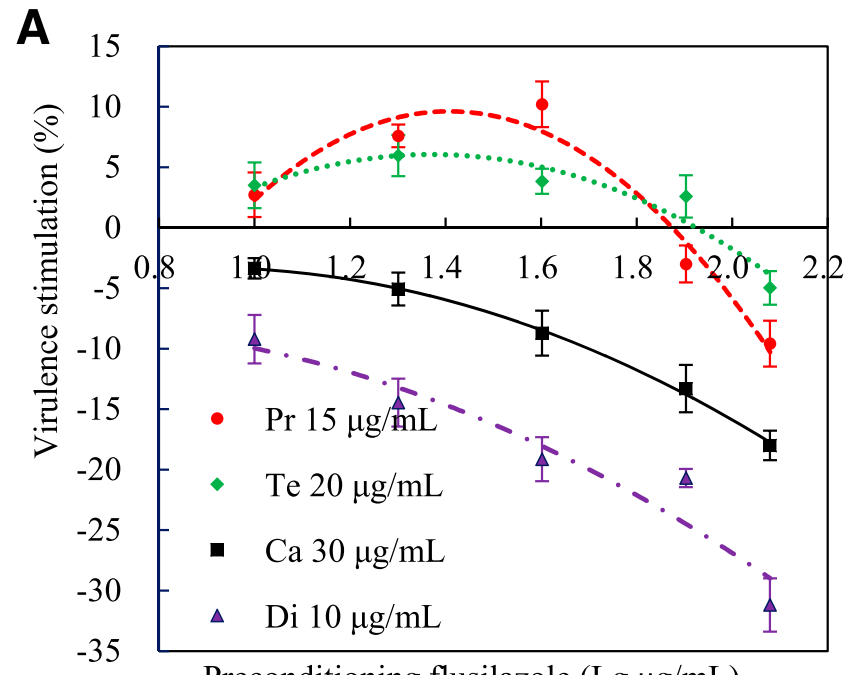

Preconditioning flusilazole $(\mathrm{Lg} \mu \mathrm{g} / \mathrm{mL})$

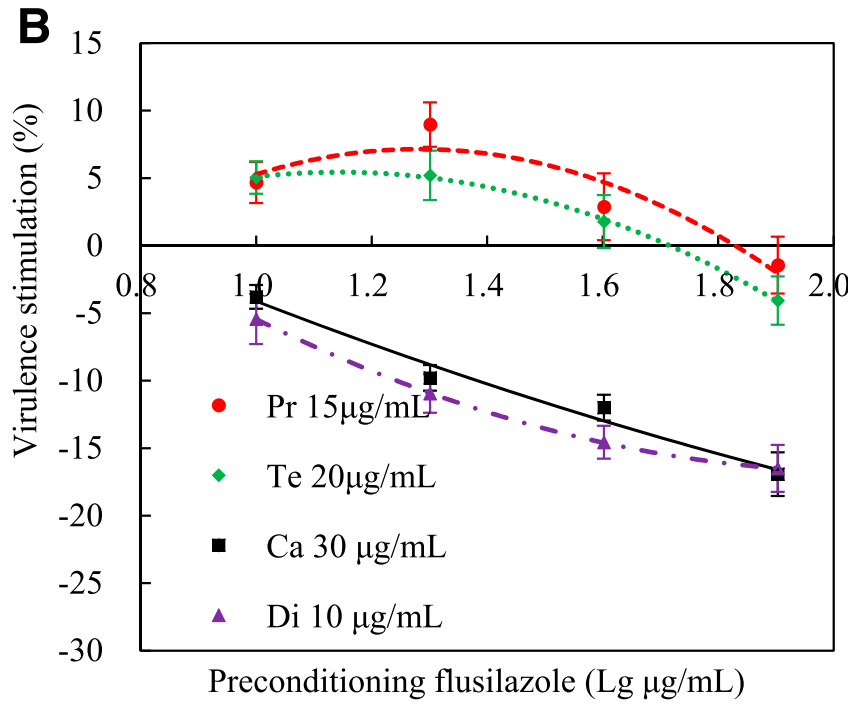

Fig. 5. Effects of preconditioning with flusilazole on virulence of Sclerotinia sclerotiorum isolates A, HN-24 and B, GS-7 on rapeseed leaves sprayed with prochloraz ( $\mathrm{Pr}$ ), tebuconazole (Te), carbendazim (Ca), or dimethachlone (Di). Error bar denotes the standard error of the mean of four repetitions. 
Preconditioning with a fungicide is likely to exert hormetic effects for subsequent exposure to a fungicide with the same MoA as that of the preconditioning fungicide, whereas no preconditioning hormetic effects could be detected for subsequent exposure to a fungicide with a different MoA.

The preconditioning stimulatory effects on mycelial growth on PDA and on virulence to rapeseed plants were calculated in the present study with the nonpreconditioned control as the denominator. Hence, the preconditioning effects were stimulatory relative to the nonpreconditioned control. Flusilazole at the preconditioning concentrations employed in the present study caused slight to obvious inhibitions on the mycelial growth and virulence of S. sclerotiorum in the first generation. For the nonpreconditioned control, later exposure to either flusilazole, prochloraz, tebuconazole, carbendazim, or dimethachlone caused substantial inhibitions on mycelial growth on PDA and on virulence to rapeseed plants. For the preconditioned mycelia, later exposure to flusilazole or other fungicides also caused inhibitions on mycelial growth and virulence compared with the nonpreconditioned control. The stimulatory effects of preconditioning treatments were, in fact, the decreases in the inhibitory effects of the subsequent exposure to the same fungicide or other fungicides with the same MoA compared with the nonpreconditioned control. This is important for understanding the preconditioning stimulatory effects in the present study.

\section{Literature Cited}

Baraldi, E., Mari, M., Chierici, E., Pondrelli, M., Bertolini, P., and Pratella, G. C. 2003. Studies on thiabendazole resistance of Penicillium expansum of pears: Pathogenic fitness and genetic characterization. Plant Pathol. 52:362-370.

Boland, G. J., and Hall, R. 1994. Index of plant hosts of Sclerotinia sclerotiorum. Can. J. Plant Pathol. 16:93-108.

Bolton, M. C., Thomma, B. P., and Nelson, B. D. 2006. Sclerotinia sclerotiorum (Lib.) de Bary: Biology and molecular traits of a cosmopolitan pathogen. Mol. Plant Pathol. 7:1-16.

Calabrese, E. J. 2013. Hormetic mechanisms. Crit. Rev. Toxicol. 43:580-606.

Calabrese, E. J. 2015a. Hormesis: Principles and applications. Homeopathy 104: 69-82.

Calabrese, E. J. 2015b. Hormesis within a mechanistic context. Homeopathy 104: 90-96.

Calabrese, E. J. 2016a. Preconditioning is hormesis part I: Documentation, doseresponse features and mechanistic foundations. Pharmacol. Res. 110:242-264.

Calabrese, E. J. 2016b. Preconditioning is hormesis part II: How the conditioning dose mediates protection: Dose optimization within temporal and mechanistic frameworks. Pharmacol. Res. 110:265-275.

Calabrese, E. J., Bachmann, K. A., Bailer, A. J., Bolger, P. M., Borak, J., Cai, L., Cedergreen, N., Cherian, M. G., Chiueh, C. C., Clarkson, T. W., Cook, R. R., Diamond, D. M., Doolittle, D. J., Dorato, M. A., Duke, S. O., Feinendegen, L., Gardner, D. E., Hart, R. W., Hastings, K. L., Hayes, A. W., Hoffmann, G. R., Ives, J. A., Jaworowski, Z., Johnson, T. E., Jonas, W. B., Kaminski, N. E.,
Keller, J. G., Klaunig, J. E., Knudsen, T. B., Kozumbo, W. J., Lettieri, T. Liu, S. Z., Maisseu, A., Maynard, K. I., Masoro, E. J., McClellan, R. O., Mehendale, H. M., Mothersill, C., Newlin, D. B., Nigg, H. N., Oehme, F. W., Phalen, R. F., Philbert, M. A., Rattan, S. I., Riviere, J. E., Rodricks, J., Sapolsky, R. M., Scott, B. R., Seymour, C., Sinclair, D. A., Smith-Sonneborn, J., Snow, E. T., Spear, L., Stevenson, D. E., Thomas, Y., Tubiana, M., Williams, G. M., and Mattson, M. P. 2007. Biological stress response terminology: Integrating the concepts of adaptive response and preconditioning stress within a hormetic dose-response framework. Toxicol. Appl. Pharmacol. 222:122-128.

Calabrese, E. J., and Blain, R. B. 2011. The hormesis database: The occurrence of hormetic dose responses in the toxicological literature. Regul. Toxicol. Pharmacol. 61:73-81.

Calabrese, E. J., and Mattson, M. P. 2017. How does hormesis impact biology, toxicology, and medicine? NPJ Aging Mech. Dis. 3: Article 13.

Deng, C., Graham, R., and Shukla, R. 2001. Detecting and estimating hormesis using a model-based approach. Hum. Ecol. Risk Assess. 7:849-866.

Di, Y. L., Cong, M. L., Zhang, R., and Zhu, F. X. 2016a. Hormetic effects of trifloxystrobin on aggressiveness of Sclerotinia sclerotiorum. Plant Dis. 100: 2113-2118.

Di, Y. L., Lu, X. M., Zhu, Z. Q., and Zhu, F. X. 2016b. Time-course of carbendazim stimulation on pathogenicity of Sclerotinia sclerotiorum indicates a direct stimulation mechanism. Plant Dis. 100:1454-1459.

Di, Y. L., Zhu, Z. Q., Lu, X. M., and Zhu, F. X. 2015. Pathogenicity stimulation of Sclerotinia sclerotiorum by subtoxic doses of carbendazim. Plant Dis. 99:1342-1346.

Flores, F. J., and Garzón, C. D. 2013. Detection and assessment of chemical hormesis on the radial growth in vitro of oomycetes and fungal plant pathogens. Dose Response 11:361-373.

Garzón, C. D., Molineros, J. E., Yanez, J. M., Flores, F. J., Jimenez-Gasco, M. M., and Moorman, G. W. 2011. Sublethal doses of mefenoxam enhance Pythium damping-off of geranium. Plant Dis. 95:1233-1238.

Lu, X. M., Zhang, R., Cong, M. L., Li, J. H., and Zhu, F. X. 2018. Stimulatory effects of flusilazole on virulence of Sclerotinia sclerotiorum. Plant Dis. 102: 197-201.

Lu, X. M., Zhu, Z. Q., Di, Y. L., and Zhu, F. X. 2015. Baseline sensitivity and toxic action of flusilazole to Sclerotinia sclerotiorum. Crop Prot. 78:92-98.

USDA. 2016. National strategic plan for the Sclerotinia research initiative integrated research for disease management in sunflower, canola, dry bean, pea $\&$ lentils and soybean. Online publication. United States Department of Agriculture (USDA). https://www.ars.usda.gov/ARSUserFiles/30000000/ WhiteMoldResearch/SI-Strategic-PLan_\%202017-2021_v1_0_Jan16.pdf

Wang, C., Qiu, L., Zhao, H., Wang, K., and Zhang, H. 2013. Dissipation dynamic and residue distribution of flusilazole in mandarin. Environ. Monit. Assess. 185:9169-9176.

Wang, Y., Hou, Y. P., Chen, C. J., and Zhou, M. G. 2014. Detection of resistance in Sclerotinia sclerotiorum to carbendazim and dimethachlon in Jiangsu Province of China. Australas. Plant Pathol. 43:307-312.

Yu, S., Qin, D., Wu, Q., Guo, X., Han, L., and Jiang, S. 2011. Residue and dissipation dynamics of flusilazole in apple and soil. Bull. Environ. Contam. Toxicol. 86:319-322.

Zhou, F., Liang, H. J., Di, Y. L., You, H., and Zhu, F. X. 2014. Stimulatory effects of sublethal doses of dimethachlon on Sclerotinia sclerotiorum. Plant Dis. 98: 1364-1370. 